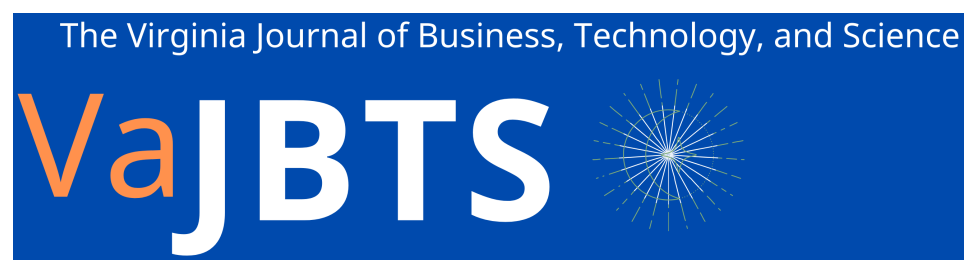

\title{
Analysis of the 2D complex Ginzburg-Landau Equation using Singular Value Decomposition
}

\author{
Edwin Ding, Ph.D. ${ }^{1}$ and Emily Gottry ${ }^{1}$ \\ ${ }^{1}$ Department of Mathematics, Physics, and Statistics, Azusa Pacific University, Azusa, CA 91702, USA
}

April 23, 2021

\begin{abstract}

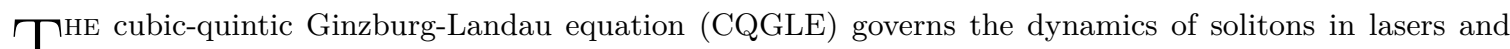
1 many optical systems. Using data obtained from the simulations of the CQGLE, we performed a singular value decomposition (SVD) to create a low dimensional model that qualitatively predicts the stability of the solitons as a function of the energy gain constant. It was found both in the full simulations and in the low dimensional model that the soliton becomes unstable when the gain exceeds a certain threshold value. Both the low dimensional model and the full simulation demonstrated the same qualitative behavior when the soliton loses stability.
\end{abstract}

\section{Introduction}

A soliton is a solitary wave that behaves like a particle in that it maintains its shape as it travels and collides. John Scott Russell was the first to study solitons after observing a solitary water wave in a canal in 1834, but it was not until the mid-1960 that other scientists and mathematicians studied solitons in depth [I]. Today, solitons have many relevant applications, including in lasers, optics, communications, ocular surgeries, and nuclear fusion [Z]. Two-dimensional spatial solitons in optical systems such as waveguide arrays are governed by the complex cubic-quintic Ginzburg-Landau equation (CQGLE). In the dimensionless form where all variables and parameters have been normalized by their respective characteristic scales, the CQGLE is:

$$
\begin{gathered}
i \frac{\partial u}{\partial t}+\frac{D}{2} \nabla^{2} u+(\gamma-i \beta)|u|^{2} u-(\nu-i \mu)|u|^{4} u=i\left(g(t)\left(1+\tau \nabla^{2}\right)-\delta\right) u, \\
g(t)=\frac{2 g_{0}}{1+\|u\|^{2} / e_{0}} .
\end{gathered}
$$

The CQGLE is a generalization of the nonlinear Schrödinger equation that includes dissipation and other optical effects $[\mathbb{Z}, \mathbf{B}] . u$, the complex amplitude of the soliton, is a function of the transverse variables, $x$ and $y$, and time, $t$. The parameter $D$ is the averaged diffraction coefficient. $\gamma$ and $\nu$ represent the cubic and quintic selfphase modulations. The time-dependent gain $g(t)$ depends on the gain constant $g_{0}$ and the total cavity energy $\|u\|^{2}=\int_{-\infty}^{\infty} \int_{-\infty}^{\infty}|u|^{2} d x d y$ which is saturated at the saturating energy $e_{0} . \tau$ determines the bandwidth of the gain. $\delta$ is the total linear loss, and $\beta$ and $\mu$ represent nonlinear losses [3].

Research Focus All of the parameters in the CQGLE (D) would impact the behavior and dynamics of the corresponding soliton. This research focused entirely on how the stability of the single soliton depends on the saturated gain $g(t)$ by changing the value of the gain constant $g_{0}$ and holding all other parameters constant. We used MATLAB to solve equation (⿴囗十) numerically, and found that at a moderate $g_{0}$ value, a single, stable soliton as seen in Figure $\mathbb{W}$ is formed from an arbitrary initial condition (e.g. white noise). At a certain higher threshold $g_{0}$, the soliton begins to become unstable, resulting in a visible pulsation. The goal of this research was to find that threshold $g_{0}$ value without running extensive full simulations, which, for the level of accuracy desired in two spatial dimensions, require days to execute. So, a low dimensional model was desired that would qualitatively reproduce the true pulse dynamics. 


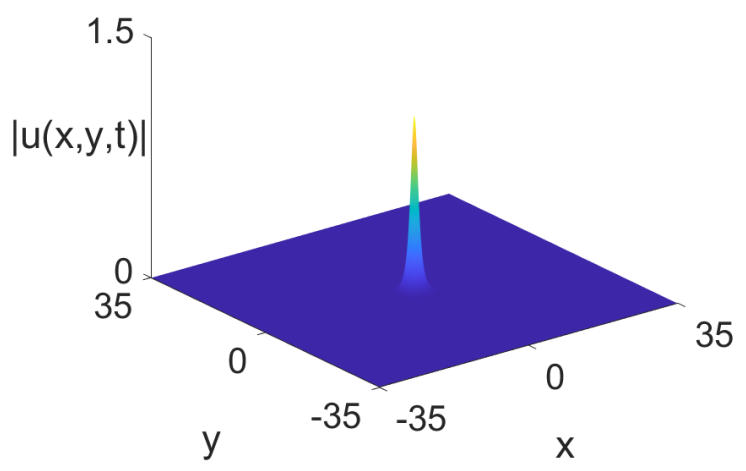

Figure 1: Stable single pulse at steady state.

\section{Singular Value Decomposition}

The most dominant modes, also known as the principal components, that contain the key characteristics of the time-evolution of a single soliton governed by the CQGLE (⿴), are the eigenvectors of the covariance matrix, $A^{*} A[Z]$, where $A$ is the data matrix that contains the numerical solution of the CQGLE corresponding to a particular set of parameters. It is well known that these dominant modes are equivalent to the singular vectors of $A$ associated with large singular values. Therefore, a singular value decomposition (SVD) was performed on $A$ to find the most dominant characteristics in the soliton evolution.

Figure 2 shows the first four modes in the SVD and their corresponding singular values. Most of the key information about the soliton dynamics is contained within the first few modes since their weights, as indicated by the singular values, were significantly greater than the other mode weights. Through experimentation, we determined that six modes was the number required to construct a low dimensional model that qualitatively reproduces the soliton dynamics. We decided to use seven modes to retain a slightly higher percentage of the information than the minimum required.

Singular Value $=178.0874$

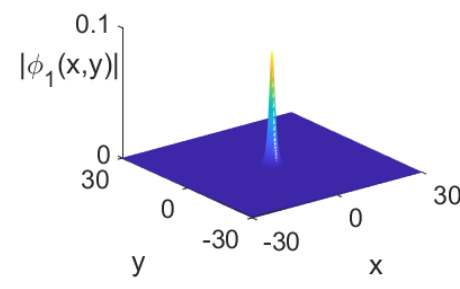

Singular Value $\mathbf{=} \mathbf{0 . 6 0 7 4}$

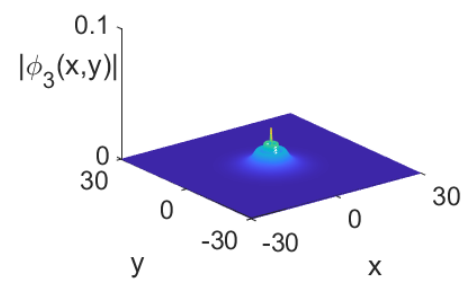

Singular Value $=3.4937$

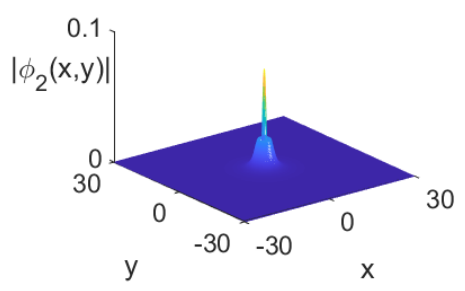

Singular Value $=\mathbf{0} 0.034025$

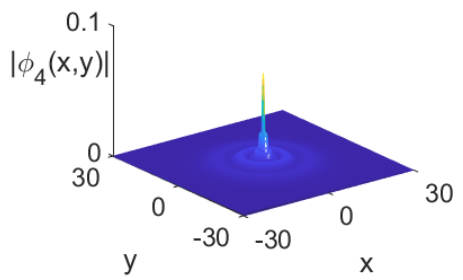

Figure 2: First four modes from a full numerical simulation of the CQGLE $\left(g_{0}=1.45, D=0.5, \gamma=1.5\right.$, $\left.\nu=0.08, e_{0}=1, \tau=0.1, \beta=0.8, \mu=0.5\right)$. The most dominant mode resembles a stable single pulse as expected. 


\section{Low Dimensional Model}

To derive a low dimensional model that describes the soliton dynamics, we expand $u(x, y, t)$ in terms of its principal components as

$$
u(x, y, t)=\sum_{n=1}^{m} a_{n}(t) \phi_{n}(x, y) .
$$

Here $a_{n}(t)$ is the time-dependent modal amplitude of the $n^{t h}$ principal component $\phi_{n}(x, y)$, and $m$ is the chosen number of modes ( 7 in our case). Plugging this into the CQGLE (⿴) and using the orthogonality of the modes, we obtain the evolution equations of the modal amplitudes

$$
a_{n}^{\prime}(t)=\left\langle F, \phi_{n}\right\rangle, n=1,2, \ldots, m
$$

Here $\langle\cdot\rangle$ denotes the inner product, in which $F$ is the right side of the CQGLE when written as $u_{t}=F$. Although the modes $\phi_{n}(x, y)$ were obtained using $g_{0}=1.45$, the above system of $m$ first-order ODE's was solved numerically for different $g_{0}$ values.

\section{Results}

Figure 3 plots the total cavity energy $E=\|u\|^{2}$ over time from full simulations of the CQGLE (四) and the low dimensional model (see equations (지) and (피)) for $g_{0}=1.7$ and $g_{0}=2.3$. The energy plots from the low dimensional model are not identical to those from the full CQGLE simulation. Although the low dimensional model is inadequate to capture the quantitative form of the periodic energy fluctuation, the qualitative behavior is accurately reproduced. At $g_{0}=1.7$, the cavity energy in both simulations stabilizes, resulting in a stable single soliton that resembles the one shown in figure $\mathrm{W}$. At a higher $g_{0}$ value of 2.3, the soliton is unstable, resulting in periodic fluctuations in the cavity energy. This kind of pulsation is also observed in the three-dimensional soliton plots for the low dimensional model and full simulation (not shown here). Using the low dimensional model, we observed that the onset of instability happened at an approximate threshold value of $g_{0}=1.72$. This is in good agreement with full numerical simulations of the CQGLE (四).

Full Simulation

$$
g_{0}=1.7
$$

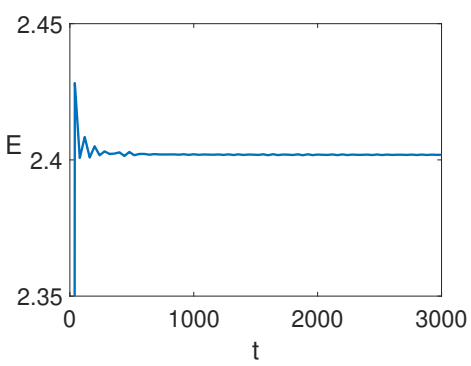

$g_{0}=2.3$

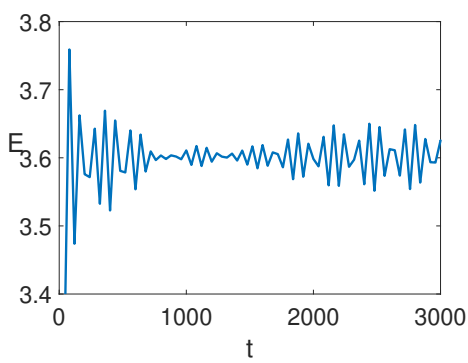

Low Dimensional Model $g_{0}=1.7$

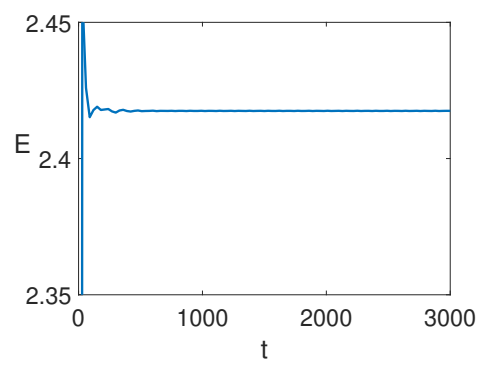

$g_{0}=2.3$

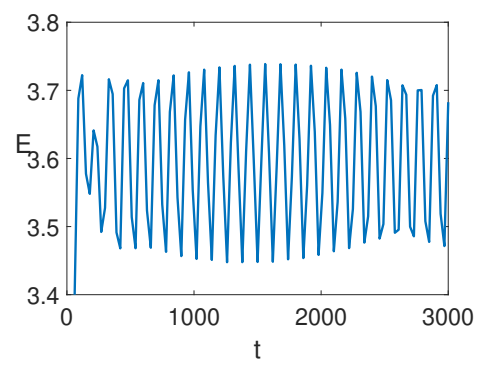

Figure 3: Evolution of the cavity energy at two $g_{0}$ values for the low dimensional model and full simulation. The qualitative behavior is the same. 


\section{Conclusion}

It is remarkable that we can qualitatively describe the soliton behavior using just seven modes for two reasons. First, the low dimensional model produces results significantly faster than a full numerical simulation of the CQGLE (四). A full simulation for even a relatively short time period requires up to a week to run in MATLAB. In contrast, the low dimensional model can qualitatively reproduce that run in less than two hours. Second, only a few modes are required, which means that one can store and use a small amount of data to keep nearly the same amount of information. The low dimensional model qualitatively describes the single soliton dynamics for a reasonable range of $g_{0}$ values without needing to preserve all the specific data. It allowed us to identify $g_{0}=1.72$ as the threshold value at which the soliton begins to lose stability and more easily study the impact of gain in $2 \mathrm{D}$ soliton behavior.

\section{References}

[1] Eilbeck, C. (2013, June 10). John Scott Russell and the Solitary Wave. Meriot-Watt University Department of Mathematics. https://www.macs.hw.ac.uk/ chris/scott_russell.html

[2] Ding, E., Shlizerman, E., \& Kutz, J. N. (2010). Modeling multipulsing transition in ring cavity lasers with proper orthogonal decomposition. Physical Review A, 82(023823), 1-10. https://journals.aps.org/pra/abstract/10.1103/PhysRevA.82.023823

[3] Ding, E., Luh, K., \& Kutz, J. N. (2011). Stability analysis of cavity solitons governed by the cubic-quintic Ginzburg-Landau equation. Journal of Physics B: Atomic, Molecular and Optical Physics, 44(065401), 1-7. https://iopscience.iop.org/article/10.1088/0953-4075/44/6/065401 